\title{
DETERMINAÇÃO DA CONDUTIVIDADE TÉRMICA EFETIVA DE FERTILIZANTES GRANULADOS: INFLUÊNCIA DA UMIDADE E DO DIÂMETRO DE PARTÍCULA
}

\author{
R. C. R. BERNARDES, J. M. CARDOSO e H. PERAZZINI \\ Universidade Federal de Itajubá, Instituto de Recursos Naturais \\ E-mail para contato: perazzini@unifei.edu.br
}

\begin{abstract}
RESUMO - O presente trabalho objetivou a determinação da condutividade térmica efetiva na estagnação de fertilizantes granulados por meio do método da sonda linear. Como etapa prévia ao estudo com fertilizantes, foi realizada uma calibração do aparato experimental com esferas de vidro. O método em regime transiente permitiu estimar a condutividade térmica na estagnação de fertilizantes granulados com diferentes tamanhos de partícula e diferentes conteúdos de umidade. Neste contexto, foi possível investigar a influência da quantidade de água e das características estruturais do meio no parâmetro efetivo de transferência de calor. Os resultados obtidos para os ensaios com o material seco se mostraram coerentes. Entretanto, os valores obtidos para o material úmido contrariaram a expetativa teórica, indicando a necessidade de investigações adicionais.
\end{abstract}

\section{INTRODUÇÃO}

A secagem está presente nos mais variados processos industriais, sendo utilizada para a remoção de voláteis dos materiais sólidos e responsável por grande parte da energia térmica empregada em um processo industrial. Sabe-se que durante a operação de secagem ocorre transferência de calor e massa dentro do leito de partículas, havendo o transporte de calor para o leito e para o interior das partículas e a consequente evaporação de água do interior das partículas e das regiões intersticiais para fora do leito. Por se tratar de um meio heterogêneo, os mecanismos de transporte envolvidos nesse processo são complexos e, logo, difíceis de serem descritos com clareza. Sendo assim, no estudo da transferência de calor, nesses casos, utiliza-se o conceito de condutividade térmica efetiva, um parâmetro que representa todos os mecanismos envolvidos e norteia o fenômeno. A dificuldade de se determinar com exatidão esse parâmetro efetivo é evidente em várias técnicas experimentais disponíveis, como se observa nos diversos resultados encontrados na literatura para um mesmo material, por exemplo. Portanto, a necessidade de se aprimorar esses métodos e consequentemente facilitar a determinação da condutividade térmica efetiva dos materiais se torna perceptível. Somado a isso, há ainda que se utilizar dos resultados provenientes das pesquisas na área para propor melhorias nos equipamentos de secagem, e produzir novas tecnologias para a indústria de fertilizantes. Dado este contexto, o presente trabalho objetiva contribuir para o estudo de secagem por meio da investigação da transferência de calor, utilizando como parâmetro principal do estudo a condutividade térmica efetiva do fertilizante. 


\section{MATERIAIS E MÉTODOS}

\subsection{O Fertilizante}

O material utilizado no procedimento experimental foi um fertilizante granulado do tipo superfosfato simples, produzido pela Heringer Fertilizantes S.A., disponível em dois diâmetros médios, $2,0 \mathrm{~mm}$ e 2,36 $\mathrm{mm}$. Para a primeira etapa do experimento utilizou-se o material seco em estufa a $105^{\circ} \mathrm{C}$. Os procedimentos foram realizados com réplica. Na segunda etapa do experimento, procurou-se verificar a influência da umidade na transferência de calor no meio. Para isso, foram utilizados dois procedimentos para umidificar a amostra. No primeiro, a amostra foi deixada imersa em água por 24 horas e por 48 horas, obtendo assim um material com 19,8 \% e 22,7 \% de umidade (base úmida), respectivamente. Para a colocação do material que estava imerso na célula experimental foi realizado um peneiramento para a retirada da água em excesso. No segundo procedimento, a amostra foi umidificada por meio da proximidade com água em estado de evaporação, sem o contato direto com a água líquida, obtendo assim uma umidade média de 12,6\% (base úmida).

\subsection{Metodologia e Aparato Experimental}

O método utilizado na determinação da condutividade térmica efetiva das amostras de fertilizante granulado foi o método da sonda linear. O aparato experimental consiste de uma célula cilíndrica de aço inox com $5 \mathrm{~cm}$ de diâmetro interno e $30 \mathrm{~cm}$ de comprimento, revestida com uma camada de $1 \mathrm{~cm}$ de espessura de poliuretano. Uma sonda linear de aço inox, contendo uma resistência bobinada homogeneamente em seu interior, contém um termopar tipo K junto à sua superfície. A Figura 1 ilustra o aparato experimental utilizado.

Figura 1 - Aparato experimental para determinação da condutividade térmica efetiva.

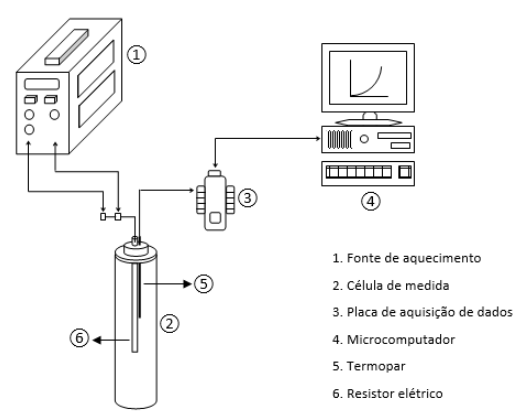

O método é fundamentado na Lei de Fourier da Condução de Calor, em regime transiente, que pode ser descrita matematicamente pela Equação (1).

$$
\nabla^{2}=\frac{1}{\alpha} \frac{\partial \mathrm{T}}{\partial \mathrm{t}}
$$

Com as condições de contorno apresentadas em seguida, resolve-se analiticamente a Equação (1), obtendo-se uma solução aproximada, descrita pela Equação (5), como mostra Souza et al. (1999). 
Condições de contorno:

i) $\quad \mathrm{t}=0$ e $\mathrm{r}>0: \mathrm{T}(\mathrm{r}, \mathrm{t})=\mathrm{T}_{0}$

ii) $\quad \mathrm{t}>0$ e $\mathrm{r} \rightarrow 0: \lim _{\mathrm{r} \rightarrow 0}\left(\mathrm{r} \frac{\partial \mathrm{T}}{\partial \mathrm{t}}\right)=-\frac{\mathrm{Q}}{2 \pi \mathrm{k}_{\text {eff }}}$

iii) $\quad \mathrm{t}>0$ e $\mathrm{r} \rightarrow \infty: \lim _{\mathrm{r} \rightarrow \infty} \Delta \mathrm{T}(\mathrm{r}, \mathrm{t})=0$

$\mathrm{k}_{\mathrm{eff}}=\frac{\mathrm{Q}}{4 \pi(\mathrm{d} \Delta \mathrm{Td} \ln \mathrm{t})}$

Em um gráfico de temperatura em função do tempo linearizado, ou ainda, $\Delta \mathrm{T} x \ln \left(\mathrm{t}_{2} / \mathrm{t}_{1}\right)$, temos uma porção linear cujo coeficiente angular corresponde a $\mathrm{Q} / 4 \pi \mathrm{k}_{\text {eff. }}$ A resolução acima requer a eliminação dos efeitos de condução e radiação de calor, além da hipótese de que geração de calor é constante ao longo do fio (longo e fino) e a consideração de que as propriedades das amostras de teste, infinitas, sejam uniformes.

\section{RESULTADOS E DISCUSSÃO}

\subsection{Calibração da Célula}

Para se obter confiabilidade nos resultados, foi realizada uma etapa prévia de calibração da célula experimental. Para isso, foram usadas esferas de vidro com diâmetro médio de $0,92 \mathrm{~mm}$, como material de referência. Nessa etapa, o procedimento de coleta dos dados de temperatura foi realizado dez vezes, mantendo o empacotamento do meio poroso constante a cada 5 corridas. As corridas tiveram duração média de 40 minutos com os dados coletados em intervalos de 20 segundos. A potência linear fornecida pela fonte de calor foi de $3,7 \mathrm{~W} \cdot \mathrm{m}^{-1}$ (fonte ajustada em: 7,4V e $0,13 \mathrm{~A}$; comprimento da sonda: $0,26 \mathrm{~m}$ ). Foram obtidos, ao todo, 10 conjuntos de dados de temperatura em função do tempo. A Figura 2 (a) mostra os dados de temperatura média do meio poroso em função do tempo linearizado, dos quais foi possível estimar a condutividade térmica efetiva na estagnação, para cada caso analisado. A Figura 2 (b) mostra a porção linear escolhida para se determinar o coeficiente angular da reta, a fim de se utilizar na Equação (5) para determinar $k_{\text {eff. }}$.

Figura 2 - Calibração da célula: (a) temperatura em função do tempo; (b) temperatura em função do tempo linearizado.
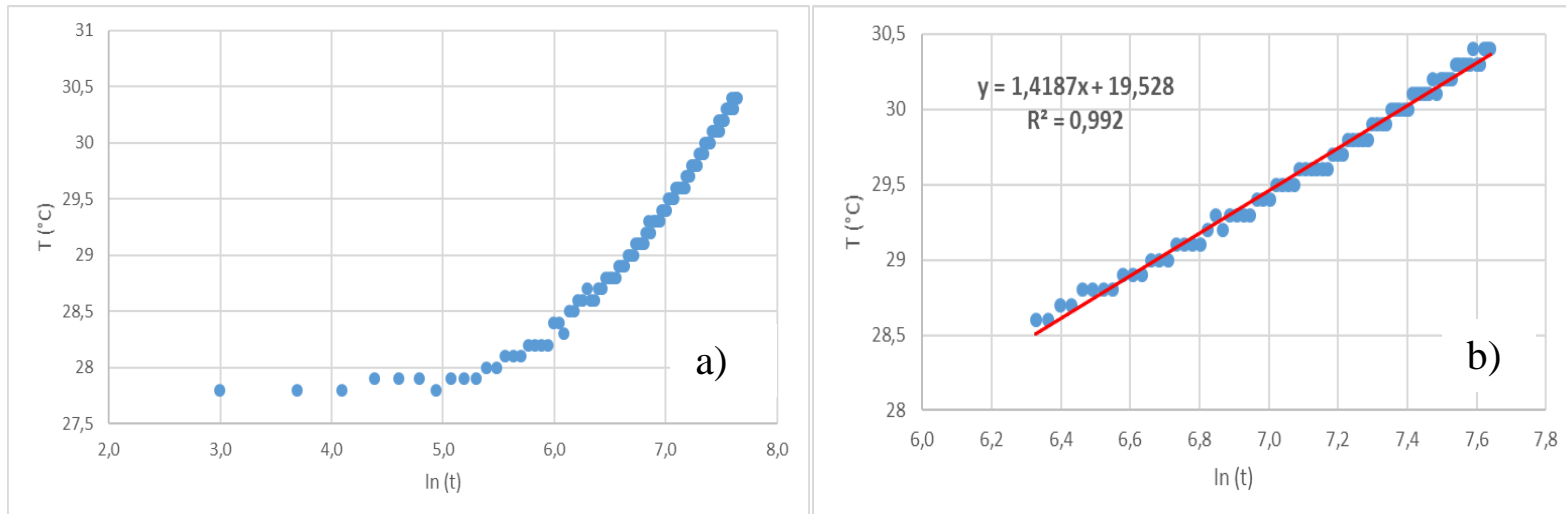
Para a condutividade térmica efetiva das esferas de vidro foi obtido um valor médio de $0,213 \mathrm{~W} \cdot \mathrm{m}^{-1} \mathrm{~K}^{-1}\left(0,030 \mathrm{cal} \cdot \mathrm{cm}^{-1} \mathrm{~min}^{-1}{ }^{\circ} \mathrm{C}^{-1}\right)$, um desvio padrão de $4,5 \%$ e uma variância de $0,2 \%$, o que mostra que os resultados são reprodutíveis. O valor obtido experimenalmente está bem próximo daquele obtido por Freire (1979), para uma potência de $35 \mathrm{~W}$, que foi de 0,033 $\mathrm{cal}^{\circ} \mathrm{cm}^{-1} \mathrm{~min}^{-1 \circ} \mathrm{C}^{-1}$ para esferas de vidro.

\subsection{Determinação da Condutividade Térmica: influência do diâmetro da partícula}

A Tabela 1 mostra os resultados obtidos do procedimento realizado com o material seco para se investigar a influência do diâmetro médio de partícula na condutividade térmica efetiva do meio.

Tabela 1 - Valores médios obtidos para a condutividade térmica efetiva do fertilizante com diferentes diâmetros de partícula.

\begin{tabular}{|c|c|c|c|c|}
\hline $\begin{array}{c}\text { Diâmetro de } \\
\text { partícula }(\mathrm{mm})\end{array}$ & $\begin{array}{c}\text { Tempo de } \\
\text { corrida }(\mathrm{h})\end{array}$ & $\mathrm{k}_{\mathrm{eff}}\left(\mathrm{W} \cdot \mathrm{m}^{-1} \mathrm{~K}^{-1}\right)$ & $\mathrm{R}^{2}$ & Desvio padrão \\
\hline \hline 2,00 & 1 & 0,398 & 0,973 & $10,7 \%$ \\
\hline 2,00 & 4 & 0,159 & 0,995 & $5,3 \%$ \\
\hline 2,36 & 1 & 0,383 & 0,965 & $4,4 \%$ \\
\hline 2,36 & 4 & 0,144 & 0,994 & $1,9 \%$ \\
\hline
\end{tabular}

Nota-se que o valor médio de $\mathrm{k}_{\text {eff }}$ obtido para as amostras com 2,0 mm de diâmetro de partícula foi maior do que o valor médio obtido para as amostras com 2,36 mm de diâmetro, podendo-se afirmar que houve coerência nos resultados. Isso pode ser explicado pelo fato de que meios porosos compostos por partículas com menores diâmetros possuem uma menor porosidade. Sendo assim, a presença de menos espaços vazios contribui para o aumento da condutividade térmica efetiva do meio. Convém ressaltar que foram obtidos diferentes valores de $k_{\text {eff }}$ para diferentes tempos de duração da corrida, podendo-se inferir que as corridas com menor duração não oferceram uma porção linear representativa para que fosse possível obter dados com confiabilidade. É válido reforçar a pequena ordem de grandeza da diferença dos valores resultantes de experimentos com diferentes diâmetros. Pode-se atribuir essa diferença, de aproximadamente $0,01 \mathrm{~W} \cdot \mathrm{m}^{-1} \mathrm{~K}^{-1}$, ao fato de que o material analisado é muito irregular, de baixa esfericidade, podendo oferecer diferentes áreas de contato entre as partículas e com a sonda, a depender da maneira em que é empacotado. A diferença entre os diâmetros de partícula também é pequena, o que pode contribuir com os resultados apresentados. Com base nestas discussões, nota-se que é difícil de se obter uma descrição bem fundamentada visto a proximidade dos resultados obtidos. Mesmo que o desvio padrão não foi muito elevado, foi verificada uma imprecisão no método utilizado, como por exemplo, a obtenção de diferentes coeficientes angulares para uma mesma corrida experimental. Constata-se, dessa forma, que o resultado final é dependente da escolha adequada do trecho linear, ficando estipulado, para este trabalho, a escolha do trecho linear que apresentava o maior coeficiente de determinação. Isso sugere a utilização de novos métodos para estimar a condutividade térmica efetiva para estudos futuros. Nesse sentido, pode-se considerar a utilização de outros métodos para a determinação da condutividade térmica efetiva de fertilizantes, como por exemplo, a célula de Dickerson (1965). 


\subsection{Determinação da Condutividade Térmica: influência da umidade}

A Tabela 2 mostra os resultados obtidos para as corridas realizadas com o material úmido, a fim de se investigar a influência que o conteúdo de umidade presente no meio exerce sobre condutividade térmica efetiva do fertilizante. Nessa estapa, foram utilizadas amostras com diâmetro médio de 2,36 mm.

Tabela 2 - Valores médios obtidos para a condutividade térmica efetiva do fertilizante em amostras com diferentes valores de umidade.

\begin{tabular}{|c|c|c|}
\hline $\begin{array}{c}\text { Umidade (base } \\
\text { úmida) }\end{array}$ & $\mathrm{k}_{\mathrm{eff}}\left(\mathrm{W} \cdot \mathrm{m}^{-1} \mathrm{~K}^{-1}\right)$ & $\begin{array}{c}\text { Tempo de } \\
\text { corrida }(\mathrm{h})\end{array}$ \\
\hline \hline Seco & 0,144 & 4 \\
\hline $12,6 \%$ & 0,356 & 1 \\
\hline $19,8 \%$ & 0,178 & 4 \\
\hline $22,7 \%$ & 0,276 & 1 \\
\hline
\end{tabular}

Sabendo-se que a condutividade térmica da água pura $\left(\mathrm{k}=0,6 \mathrm{~W} \cdot \mathrm{m}^{-1} \mathrm{~K}^{-1}\right)$ é maior do que a do ar $\left(\mathrm{k}=0,025 \mathrm{~W} \cdot \mathrm{m}^{-1} \mathrm{~K}^{-1}\right)(\mathrm{KEEY}, 1992)$, à medida que se aumentava a quantidade de água presente nos poros das partículas, ou seja, aumentando-se da umidade do material, esperava-se que a condutividade térmica efetiva do mesmo também se elevasse. No entanto, esse resultado não foi observado, como fica evidente na Tabela 2. Nota-se também que o tempo de corrida para o fertilizante úmido ocasiona uma diferença significativa no resultado final obtido. Estes fatos podem ser explicados analisando-se os resultdos apresentados na Figur 3. A Figura 3, mostra as curvas características de corridas realizadas com o material fertilizante, seco e úmido, para efeitos de comparação e tentativa de se esclarecer o motivo do resultado obtido não estar de acordo com o a literatura.

Figura 3 - Temperatura em função do tempo para amostras seca e úmida.

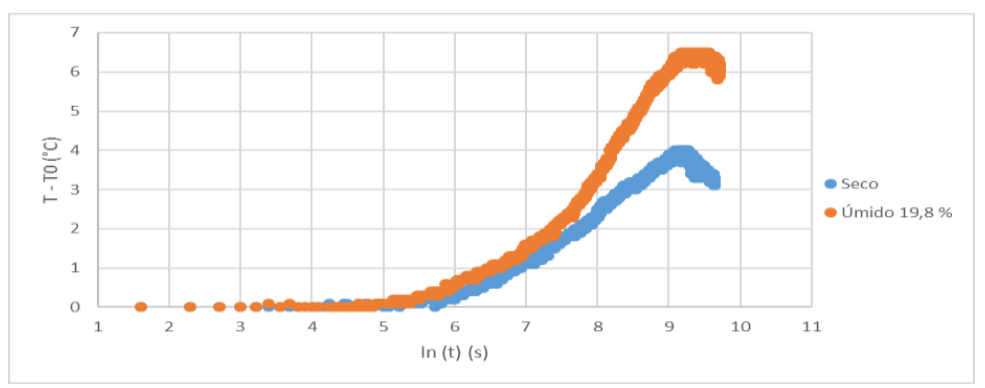

Além de atribuir os resultados a erros experimentais, uma hipótese pode ser feita. Como mostra a Equação (5), o coeficiente angular da porção linear obtida das curvas de temperatura em função do tempo, encontra-se no denominador da fração usada no cálculo da condutividade térmica efetiva. Observando a Figura 3, nota-se que a inclinação da curva para o material úmido, é sempre maior do que a inclinação da curva característica do material seco. Sendo assim, o coeficiente angular da porção linear referente ao fertilizante úmido sempre será maior do que o coeficiente angular referente ao fertilizante seco. Consequentemente, a condutividade térmica efetiva do meio úmido será sempre menor do que do meio seco. Possivelmente, a potência utilizada e o tempo longo de corrida $(4 \mathrm{~h})$ possibilitou a evaporação de uma quantidade significativa de água, contribuindo om os resultados apresentados. 


\section{CONCLUSÕES}

Neste trabalho, foi realizado um estudo da transferência de calor visando a determinação da condutividade térmica efetiva na estagnação de um fertilizante granulado, sendo analisada a influência do conteúdo de umidade e do diâmetro de partícula. Com base nos resultados obtidos, pode-se afirmar que a sonda utilizada no procedimento experimental oferece reprodutibilidade aos resultados. No entanto, nos ensaios realizados com o fertilizante ficou evidente a necessidade de se ampliar o número de corridas com as amostras para se obter resultados mais precisos. Nota-se ainda que, dado o fato de que o método se mostrou um tanto impreciso, é válida a realização de testes com outros métodos para fins de comparação. O tamanho da partícula se mostrou pouco influente na condutividade térmica. A influência da umidade, por sua vez, mostrou-se ao contrário do esperado. Torna-se evidente a importância dos estudos na área de fenômenos de transporte, uma vez que, os resultados obtidos mostraram que um procedimento experimental de caráter inicialmente simples, pode apresentar resultados inesperados, dificultando assim, interpretações acerca dos fenômenos envolvidos. No contexto de engenharia, pode-se dizer que qualquer progresso na área se torna válido e necessário para otimizar processos industriais, especialmente o processo de secagem, processo este que está estritamente relacionado aos fenômenos de transferência de calor que ocorrem no meio, caracterizados pela condutividade térmica efetiva.

\section{NOMENCLATURA}

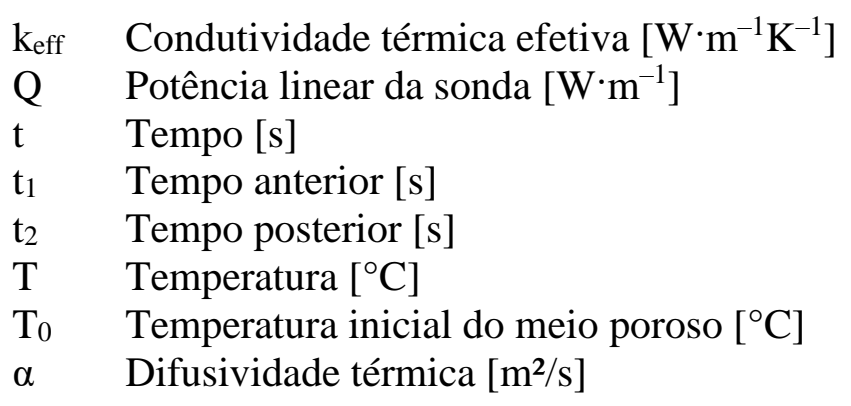

\section{REFERÊNCIAS}

DICKERSON, R.W. An aparatus for the measurement of thermal diffusivity of foods. Food Tech., v. 5, p. 198-204, 1965.

FREIRE, J. T. Transferência de calor em meios porosos. 1979. 223p. Tese (Doutorado em Engenharia Química) - COPPE, Universidade Federal do Rio de Janeiro, Rio de Janeiro, 1979.

KEEY, R. B. Drying of loose and particulate materials. New York: Hemisphere Publishing Corporation, 1992.

PIETROBON, C. L. R. Desenvolvimento de sistemas para a determinação da condutividade térmica. 1988. 122p. Dissertação (Mestrado em Engenharia Química) - PPGEQ, Universidade Federal de São Carlos, São Carlos, 1988.

SOUZA, R.; PIMENTEL, L. C. G.; ORLANDE, H. R. B. Determinação da condutividade térmica utilizando o método da sonda linear. In: congresso brasileiro de engenharia mecânica, 15. 1999, Águas de Lindóia. 